\title{
Measurement of Atomic Positions at Twin Boundaries by HREM
}

\author{
F.C. Lovey * and A.M. Condó** \\ * Centro Atómico Bariloche, 8400 Bariloche, Argentina \\ ** Also at Consejo Nacional de Investigaciones Científicas y Técnicas of Argentina
}

Twinned microstructures are observed in a large number of materials. In some cases they are related to the phase transformations, like the martensitic transformation. In other cases twinning is an important mechanism for the plastic deformation and the mechanical properties of numerous metals and alloys. In all these cases the knowledge of the atomic structure at the twin boundaries is of great interest. High resolution electron microscopy (HREM) coupled with image simulations is one of the most suitable techniques for studying twin interfaces.

As an example, a HREM image of an important twin interface, appearing in the $\beta \rightarrow 2 \mathrm{H}$ martensitic transformation in $\mathrm{Cu}-\mathrm{Zn}-\mathrm{Al}$ alloys [1], is shown in figure 1a. The interface is parallel to the (121) planes; this is called type I twinning. It can be deduced from figure 1a that the interface shows an atomic configuration with mirror antisymmetry. The mirror plane coincides with an atomic plane which is shared by the twinned variants.

In order to interpret the interface structure, several models were proposed and the corresponding HREM images were simulated. In the first one, the atomic columns being at the same level in the A type planes of both variants are brought into coincidence at the boundary. Consequently the atomic columns of the B type planes of both twinned variants do not match at the interface, thus, common columns located at a half way position are suggested. A second possible model consists in bringing into coincidence the B type columns at the boundary. In this case a more open interface is obtained. In the third model no coincident site lattice appears at the interface, figure $1 \mathrm{~b}$. The distance between the (121) planes remains unchanged, thus, the mean atomic volume is preserved at the boundary. The projected supercell for image simulation corresponding to the third model is given in figure 1c. The images were simulated using the multislice method in the EMS package [2]. A simulated thickness/defocus matrix for the interface in figure $1 \mathrm{c}$ is shown in figure 2.

The distance between the $(\overline{1} 21)$ planes across the interface was measured in the experimental and simulated images of the different models. The mean intensity profile along a horizontal line, with thickness equal to the whole image gives peaks centered at the image positions of the (121) planes. From these positions, the separation of the planes was measured. The best matching with the experimental results was obtained for the interface configuration shown in figure 1c. The effect of image filtering and size of the supercell were carefully evaluated in order to obtain reliable results.

\section{References}

[1] A.M. Condó, F.C. Lovey and V. Torra, Phil. Mag. A, 2003. In press.

[2] P.A. Stadelmann, Ultramicroscopy, 21 (1987) 131. 


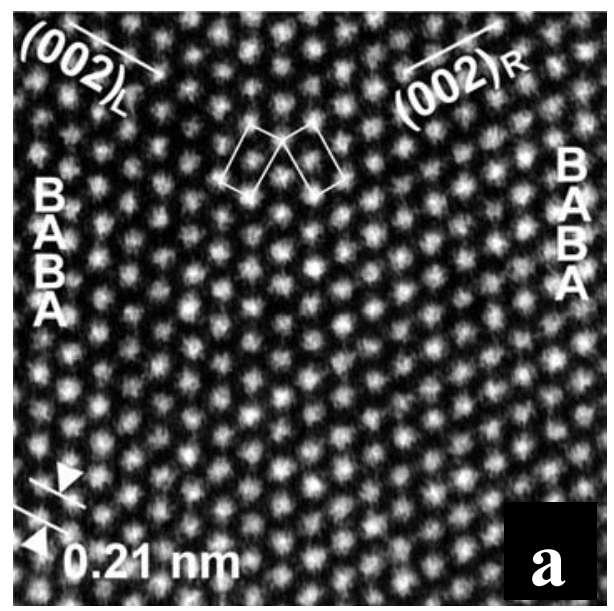

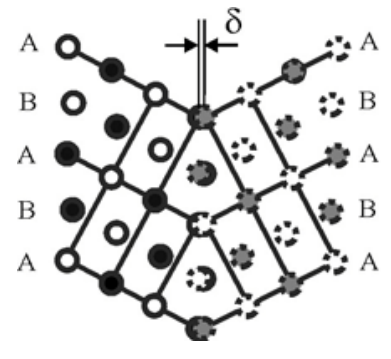

b

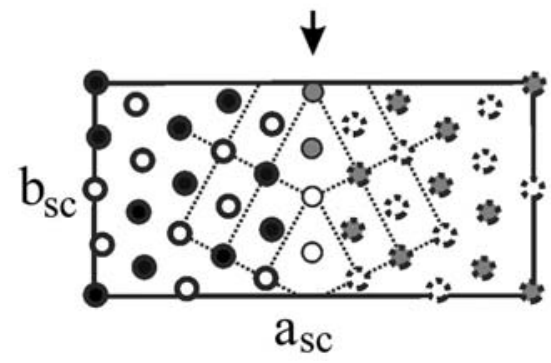

c

Figure 1: (121) twin interface in $2 \mathrm{H}$ martensite of a $\mathrm{Cu}-\mathrm{Zn}-\mathrm{Al}$ alloy. (a) Experimental HREM image observed from the $<210\rangle$ direction. The (002) basal plane stacking sequences are indicated for the variants on both sides of the interface. (b) A model for the atomic configuration at the interface, $\delta=0.0262 \mathrm{~nm}$. (c) Supercell for image simulation with lattice parameters $\mathrm{a}_{\mathrm{SC}}=2.0505 \mathrm{~nm}$ and $\mathrm{b}_{\mathrm{SC}}=0.9758 \mathrm{~nm}$.

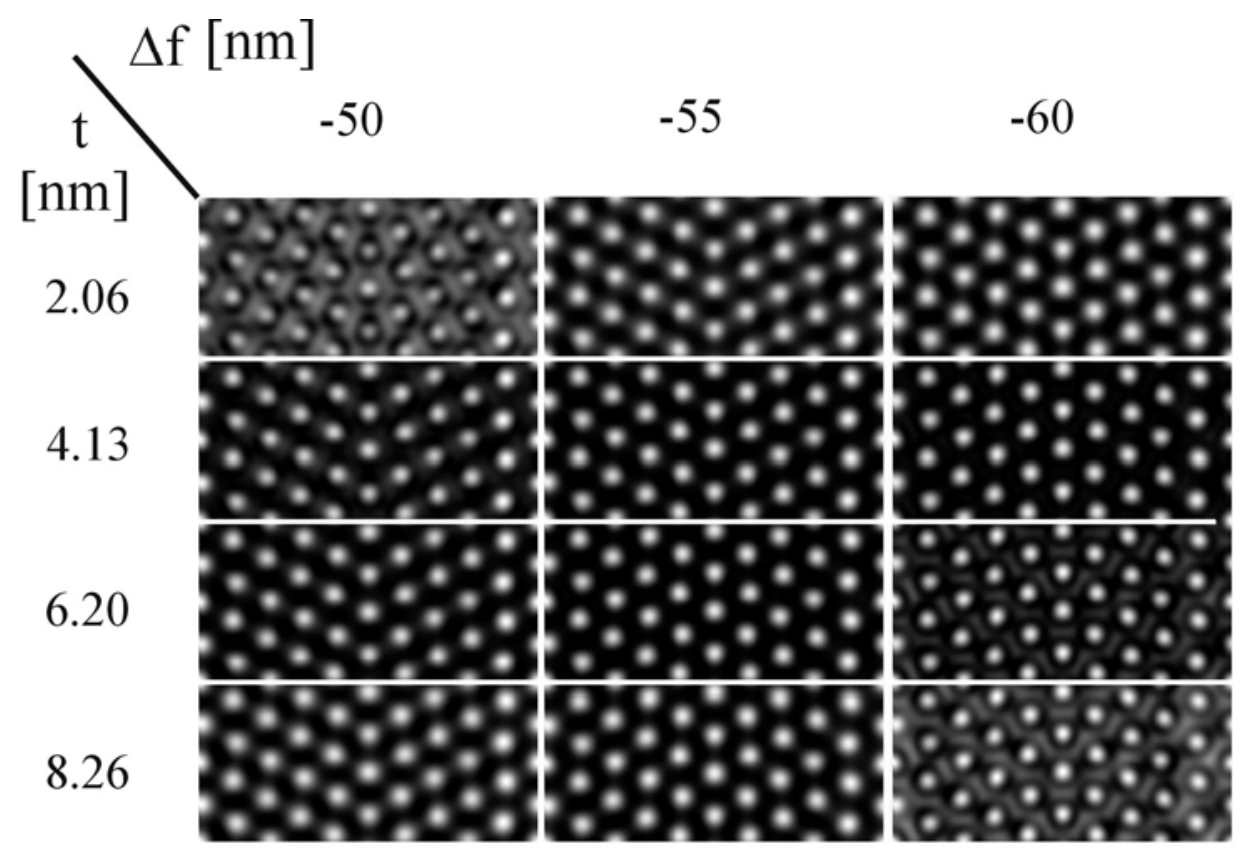

Figure 2: Thickness $(\mathrm{t})$ and defocus $(\Delta \mathrm{f})$ matrix of simulated images of the twin interface corresponding to the supercell in figure $1 \mathrm{c}$. The spherical aberration coefficient of the objective lens is $\mathrm{C}_{\mathrm{s}}=0.5 \mathrm{~mm}$. 